\title{
Effects of arginine-glycine-aspartic acid peptide- conjugated quantum dots-induced photodynamic therapy on pancreatic carcinoma in vivo
}

This article was published in the following Dove Press journal:

International Journal of Nanomedicine

5 April 2017

Number of times this article has been viewed

\author{
Ming-Ming Li* \\ jia Cao* \\ Jia-Chun Yang \\ Yu-Jie Shen \\ Xiao-Lei Cai \\ Yuan-Wen Chen \\ Chun-Ying Qu \\ Yi Zhang \\ Feng Shen \\ Lei-Ming $\mathrm{Xu}$
}

Department of Gastroenterology, Xinhua Hospital, School of Medicine, Shanghai Jiaotong University, Shanghai, People's Republic of China

*These authors contributed equally to this work

Correspondence: Lei-Ming Xu Department of Gastroenterology, Xinhua Hospital, School of Medicine, Shanghai Jiaotong University, 1665 Kongjiang Road, Yangpu District, Shanghai 200092,

People's Republic of China

Tel/fax +86 2I 25078999

Email leiming.xu@aliyun.com
Abstract: Quantum dots (QDs) conjugated with integrin antagonist arginine-glycine-aspartic acid (RGD) peptides (QDs-RGD) are novel nanomaterials with a unique optical property: a high molar extinction coefficient. Previously, we have shown that QDs-RGD demonstrate a photodynamic therapy (PDT) effect as new photosensitizers for the pancreatic cancer cell line SW1990 in vitro. Here, we investigate the application of QDs-RGD in mice bearing pancreatic tumors using PDT. To ensure that more photosensitizers accumulated in tumors, QDs-RGD were injected intratumorally. After selection of an adequate dosage for injection from analyses of biodistribution images captured by an IVIS system, PDT was initiated. Three groups were created according to different PDT procedures. In group 1, mice were injected with QDs-RGD intratumorally, and an optical fiber connected to a laser light was inserted directly into the tumor. Irradiation was sustained for $20 \mathrm{~min}$ with a laser light $(630 \mathrm{~nm})$ at $100 \mathrm{~mW} / \mathrm{cm}^{2}$. In group 2, the laser optical fiber was placed around, and not inserted into, tumors. In group 3, PDT was conducted as in group 1 but without injection of QDs-RGD. After 28 days of observation, tumors on the back of mice in group 1 grew slowly $\left(\mathrm{V} / \mathrm{V}_{0}=3.24 \pm 0.70\right)$ compared with the control groups, whose tumors grew quickly, and the mean $\mathrm{V} / \mathrm{V}_{0}$ reached $6.08 \pm 0.50$ (group 2) and $7.25 \pm 0.82$ (group 3). Histology of tumor tissues showed more necrotic tissues, more inflammatory cells, and less vascular tissue in the PDT group than those in the control groups. These results suggest that QDs-RGD-mediated PDT, with illumination using an optical fiber inserted directly into the tumor, can inhibit the growth of SW1990 tumors with high efficiency in nude mice.

Keywords: quantum dots, RGD peptides, pancreatic neoplasm, intratumoral injection, photodynamic therapy

\section{Introduction}

Pancreatic neoplasms are deadly solid malignancies. ${ }^{1,2}$ In the USA, pancreatic neoplasms are the fourth leading cause of cancer-related death, with a mean survival of $<1$ year after diagnosis., ${ }^{2,3}$ To date, surgery has been the only curative treatment, but only $20 \%$ of patients are candidates for resection. With most patients diagnosed at advanced stages, the prevalence of successful resection and 5-year survival are low. ${ }^{4,5}$ For palliative chemotherapy and/or radiotherapy, the overall prognosis is poor because of the characteristics of local invasion, early metastasis, and chemoresistance. ${ }^{6-8}$ It is important to study and improve locoregional treatments as part of a multimodal approach to the management of local pancreatic cancer. ${ }^{9}$

Photodynamic therapy (PDT) is a way of producing localized tissue necrosis and apoptosis of cancer cells. PDT is a minimally invasive treatment that damages target cells by imparting toxicity through generation of reactive oxygen species (ROS). ${ }^{10-17}$ 
Photosensitizers, oxygen, and light are the three most important elements of PDT. ${ }^{18-20}$ Selection of an appropriate photosensitizer is of paramount importance for PDT.

Quantum dots (QDs) are a class of novel nanomaterials and have been used widely in medical research. ${ }^{21-23}$ They are nanocrystals consisting of elements belonging to groups II-VI or groups III-V with diameters of $2-10 \mathrm{~nm} .{ }^{24}$ QDs possess several characteristics such as large absorption spectra, narrow and symmetric emission bands, and a high molar extinction coefficient. QDs have been used increasingly as potential photosensitizers in cellular and molecular tracing, tumor imaging in vivo, drug monitoring, and PDT. ${ }^{25,26}$ Furthermore, QDs can be conjugated with various ligands, antibodies, or peptides to prepare functional nanoparticle probes with unique properties. ${ }^{27-29}$

The arginine-glycine-aspartic acid (RGD) peptide sequence is an integrin antagonist that can link to integrin $\alpha v \beta 3$. Integrin plays a critical part in the regulation of the growth, metastasis, and angiogenesis of tumors. ${ }^{30-32}$ Therefore, creation of RGD-conjugated quantum dots (QDs-RGD) can help to trace and image tumor cells in vivo.

Previously, we demonstrated the potential applications of QDs-RGD as photosensitizers in the PDT of pancreatic cancer cells in vitro. ${ }^{33,34}$ With illumination, conduction band electrons can be transferred to surrounding oxygen molecules and produce ROS. Furthermore, our studies have suggested that with an adequate dosage of nanomaterials, there would be a lower toxic effect on pancreatic cancer cells without illumination but that prominent PDT effects would be elicited by illumination with green-blue light. QDs-RGD could be photosensitizers by inhibition of cell proliferation and induction of necrosis and apoptosis through ROS generation.

In this study, we constructed QDs-RGD and investigated their application in mice bearing the pancreatic cancer cell line SW1990 for verification of their use as potential photosensitizers in vivo. To ensure that more QDs-RGD accumulated in tumors, we administered QDs-RGD by intratumoral injection. Compared with conventional intravenous injection, the dosages tested in this administration might be much less. Furthermore, more QDs-RGD would approach tumors compared with conventional intravenous injection. ${ }^{35-37}$ Upon subsequent PDT, the irradiation procedure was another parameter to be re-evaluated. If the procedure of laser light illumination was conducted much closer to the tumor cells (eg, in the tumor mass), the PDT effect might be better than the irradiation method used previously. We introduced a novel irradiation procedure in which an optical fiber connected to a laser light was inserted directly into the tumor. For evaluation of physical damage to the tumor, some control groups were created for in-depth analyses. Our data suggested that QDs-RGD may become promising candidates for PDT of pancreatic cancer.

\section{Materials and methods Materials}

QDs705 are made of cadmium selenide cores with a zinc sulfide shell and a carboxylate-derivatized outer coating and were purchased from Invitrogen (Carlsbad, CA, USA). Cyclic RGD peptide was obtained from GL Biochem (Shanghai, China).

\section{Preparation of QDs-RGD}

QDs705 and RGD were conjugated to prepare QDs-RGD according to the manufacturer's instructions described in the Qdot ${ }^{\circledR}$ Antibody Conjugation kit (Life Technologies, Carlsbad, CA, USA). Borate buffer solution (20 mM, $\mathrm{pH}$ 7.4) was added to QDs705 (8 $\mu \mathrm{M}, \mathrm{pH} 9.0)$, until the final concentration was $1 \mu \mathrm{M}$. RGD peptide powder was dissolved in the buffer solution ( $20 \mathrm{mM}$ borate, $\mathrm{pH} 7.4$ ), until the concentration was $10 \mathrm{mg} / \mathrm{mL}$. The crosslinking reagent $N$-ethyl- $N^{\prime}$-dimethylaminopropyl-carbodiimide (EDC) was used to conjugate the QDs and peptides. The total molar ratio of QDs, EDC, and RGD peptides was 1:2,000:1,000. After $2 \mathrm{~h}$, the resulting molecules were eluted by a desalinating column. An eluate exhibiting fluorescence was collected and passed through ultrafiltration devices immediately to remove unreactive RGD peptides or other impurities. Only molecules of molecular weight $>50 \mathrm{kDa}$ were collected. The final concentration of QDs-RGD was $8 \mu \mathrm{M}$ solution in $50 \mathrm{mM}$ borate $(\mathrm{pH} \mathrm{8.4)}$. The resulting dispersions were obtained for further characterizations.

\section{Characterizations of QDs-RGD}

The nanoparticle size was measured based on dynamic light scattering (DLS) using a Zetasizer (NanoZS; Malvern Instruments, Malvern, UK). The size and morphology of the nanoparticles were measured by a transmission electron microscopy (TEM) using transmission electron microscope (H-600; Hitachi, Tokyo, Japan). The optical absorption spectra of QDs-RGD and QDs were recorded by a UV-visible spectrophotometer (UV-2550; Shimadzu, Kyoto, Japan) and a fluorescence spectrophotometer (LS-55; PerkinElmer, Waltham, MA, USA).

\section{Cell culture}

The human pancreatic cancer cell line SW1990 was purchased from the cell bank of the Type Culture Collection of the Chinese Academy of Sciences (Shanghai, China). 
SW1990 cells were cultured in RPMI 1640 media with $10 \%$ fetal bovine serum (FBS), $100 \mathrm{U} / \mathrm{mL}$ penicillin, and $100 \mu \mathrm{g} / \mathrm{mL}$ streptomycin in humidified air containing $5 \%$ $\mathrm{CO}_{2}$ at $37^{\circ} \mathrm{C}$. Cells in the exponential growth phase were used in subsequent experiments.

\section{Experimental animals and tumor models}

All experimental procedures were approved by the ethics committee of Xin Hua Hospital Affiliated to Shanghai Jiao Tong University School of Medicine (Shanghai, China) and with approved institutional protocols set by the China Association of Laboratory Animal Care. Specific pathogen-free female mice (BALB/c; 5 weeks; 17-20 g) were purchased from the Shanghai Laboratory Animal Center of the Chinese Academy of Sciences. They were reared under constant temperature $\left(22^{\circ} \mathrm{C} \pm 2^{\circ} \mathrm{C}\right)$ and humidity $(60 \% \pm 10 \%)$ with free access to water and mouse chow. Mice were anesthetized, and SW1990 cells $\left(5 \times 10^{6}\right)$ were injected (subcutaneously [sc]) with $200 \mu \mathrm{L}$ phosphate-buffered saline (PBS) onto the back. These mice were used for assessment of the PDT effect using QDs-RGD when tumor sizes reached 55-100 $\mathrm{mm}^{3}$.

\section{Imaging of QDs-RGD by intratumoral injection in vivo}

For whole-animal biophotonic imaging and PDT in vivo, the tumor-bearing nude mice were injected with QDs-RGD $(0.2,1,2.5,5,7.5,10$, and $25 \mathrm{pmol})$ intratumorally. The nanomaterials were injected gently using a 29 gauge needle and $0.1 \mathrm{~mL}$ syringe in the center of the tumor. Images were captured 1, 2, 3, and $5 \mathrm{~h}$ after injection for imaging and biodistribution analyses using an IVIS Lumina XRMS III imaging system (PerkinElmer).

\section{PDT in vivo}

For PDT in vivo, tumor-bearing mice were divided into three groups of three. In group 1, mice were injected with QDs-RGD intratumorally and an optical fiber connected to a laser light inserted directly into the center of the tumor. The irradiation was sustained for $20 \mathrm{~min}$ with laser light $(630 \mathrm{~nm})$ at a power density of $100 \mathrm{~mW} / \mathrm{cm}^{2}$. Actually, when we conducted the illumination, the optical fiber was inserted directly into the center of the tumor, thereby avoiding any bias caused by use of different distances. In group 2, mice received an injection of QDs-RGD at the same dose, but the laser optical fiber was placed around, and not inserted into, the tumor. In group 3, PDT was conducted the same as for group 1 but without injection of QDs-RGD. Groups 2 and 3 were used as control groups. Tumor sizes were measured using a caliper on alternate days. All the procedures described earlier were undertaken again on day 14. Tumor volumes were measured every week. The volume $\left(\mathrm{mm}^{3}\right)$ of tumors was calculated as (tumor length) $\times(\text { tumor width })^{2} / 2$. Relative tumor volume was calculated as $\mathrm{V} / \mathrm{V}_{0}$ (where $\mathrm{V}_{0}$ was the corresponding tumor volume when the treatment was initiated).

\section{Histology}

Mice were sacrificed on day 28, and histology of tumor tissues was done. Tumor tissues were separated and embedded in paraffin. Sliced tumor tissues were stained with hematoxylin and eosin (HE) and terminal deoxynucleotidyl transferase-mediated deoxyuridine triphosphate-biotin nick end labeling (TUNEL) for apoptosis analyses. Tissue sections were observed by a light microscope (DMI4000 B; Leica Microsystems, Wetzlar, Germany).

\section{Results \\ Preparation and characterization of QDs-RGD}

A representative TEM image of QDs-RGD is shown in Figure 1A: a spherical shape with a particle diameter of $\approx 10 \mathrm{~nm}$ can be seen. Figure $1 \mathrm{~B}$ shows a UV-Vis and photoluminescence spectra of a QDs-RGD sample. QDs-RGD had a luminescence emission peak at $702 \mathrm{~nm}$. A particle size analysis obtained from DLS showed that most QD particles had a diameter of $10.77 \pm 7.26 \mathrm{~nm}$ (Figure 1C), whereas QDs-RGD particles possessed a diameter of $26.36 \pm 12.59 \mathrm{~nm}$. The size of QDs-RGD using DLS was $26.36 \mathrm{~nm}$, distinctly larger than that of QDs $(10.77 \mathrm{~nm})$ (Figure 1C), but, according to TEM (Figure 1A), QDs-RGD exhibited similar diameters to those of QDs $(\approx 10 \mathrm{~nm})$. This difference in diameters measured by DLS and TEM can be attributed to the different surface states of the samples under test conditions. In brief, the samples are tested directly in an aqueous phase for DLS measurements, whereas, for TEM characterization, the water in QDs-RGD samples must be removed. Organic molecules such as RGD can be detected readily by DLS but cannot be observed using TEM. Consequently, the hydrodynamic diameter of QDs-RGD is obviously larger than that shown by TEM.

\section{Distribution of QDs-RGD by intratumoral injection in vivo}

To investigate the capability of fluorescence imaging of QDs-RGD in vivo, tumor-bearing nude mice were injected with QDs-RGD $(0.2,1,5$, and 25 pmol) intratumorally. These animals were imaged using an IVIS Lumina XRMS III imaging system $1,2,3$, and $5 \mathrm{~h}$ after injection. When the dosage was 0.2 or 1 pmol, a fluorescence signal could not 

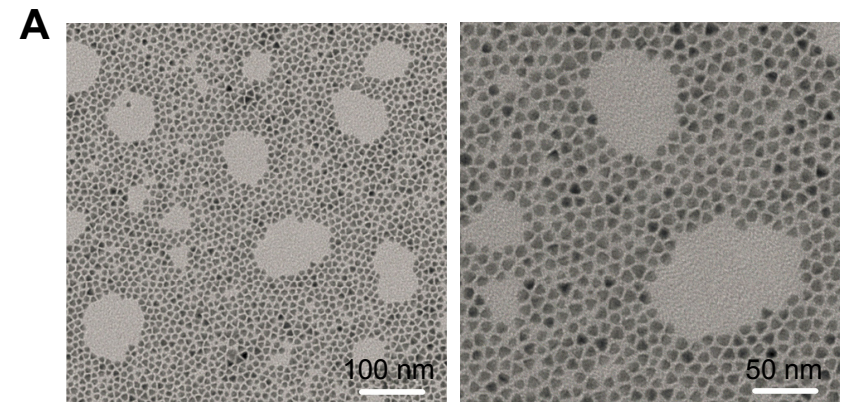

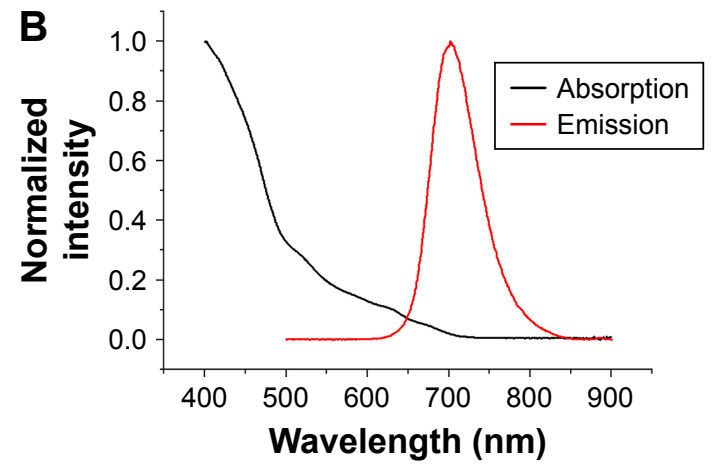

C

QDs

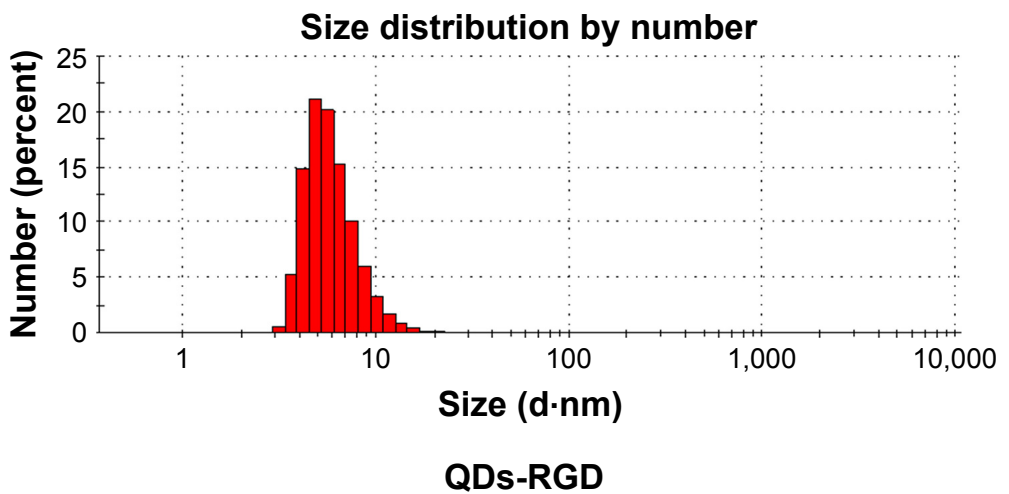

Size distribution by number

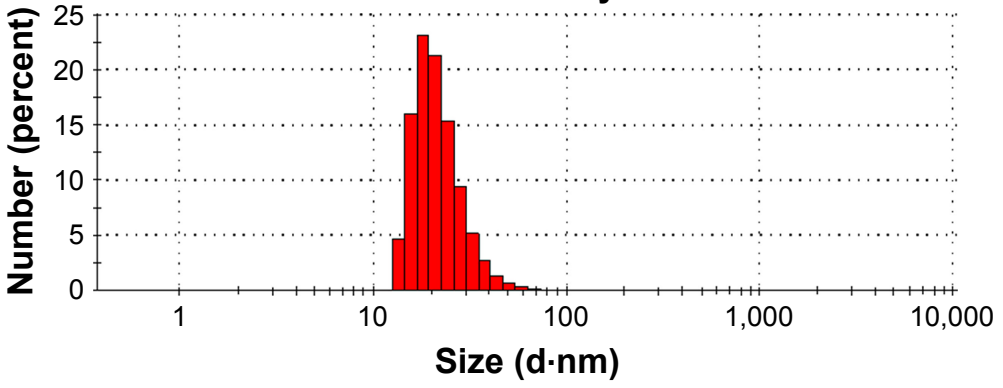

Figure I Characteristics of nanomaterials.

Notes: (A) TEM images of QDs-RGD, scale bar: left, $100 \mathrm{~nm}$; right, $50 \mathrm{~nm}$; (B) UV-PL spectra of QDs-RGD. (C) DLS measurements of QDs and QDs-RGD. QD particles possess diameters in the range of $10.77 \pm 7.26 \mathrm{~nm}$. QDs-RGD particles possess diameters in the range of 26.36 $\pm 12.59 \mathrm{~nm}$. The QDs-RGD had a luminescence emission peak at $702 \mathrm{~nm}$.

Abbreviations: DLS, dynamic light scattering; PL, photoluminescence; QDs, quantum dots; QDs-RGD, QDs conjugated with arginine-glycine-aspartic acid peptide sequence; RGD, arginine-glycine-aspartic acid; TEM, transmission electron microscopy; UV, ultraviolet.

be detected clearly (Figure 2). However, when the dosage reached 5 pmol, signals could be detected clearly from the tumor in the mouse and showed its best fluorescence effect within $1 \mathrm{~h}$. The injection sites showed much higher fluorescence intensity than the background signal produced by mouse skin. The fluorescence intensity of the tumor area decreased slowly as time passed. When the dosage reached $25 \mathrm{pmol}$, the outline of the tumor area was filled with strong signals and could not be depicted clearly. For better understanding and evaluation of the in vivo image in the tumor area, the dosages of $2.5,5,7.5$, and $10 \mathrm{pmol}$, which were distributed around the dosage of 5 pmol, were included in the subsequent biodistribution experiment. One hour after injection, the fluorescence signals in the 5, 7.5, and $10 \mathrm{pmol}$ groups were nearly identical, whereas those in the $2.5 \mathrm{pmol}$ group decreased (Figure 3).

\section{PDT in vivo}

The performance of QDs-RGD for PDT in vivo was evaluated using female BALB/nu mice with subcutaneous pancreatic cancer xenografts. Figure 4A shows the laser optical fiber used in the PDT procedure. The material was kindly donated by XINGDA Photodynamic Company (Guilin, China). To obtain better effects of illumination, 

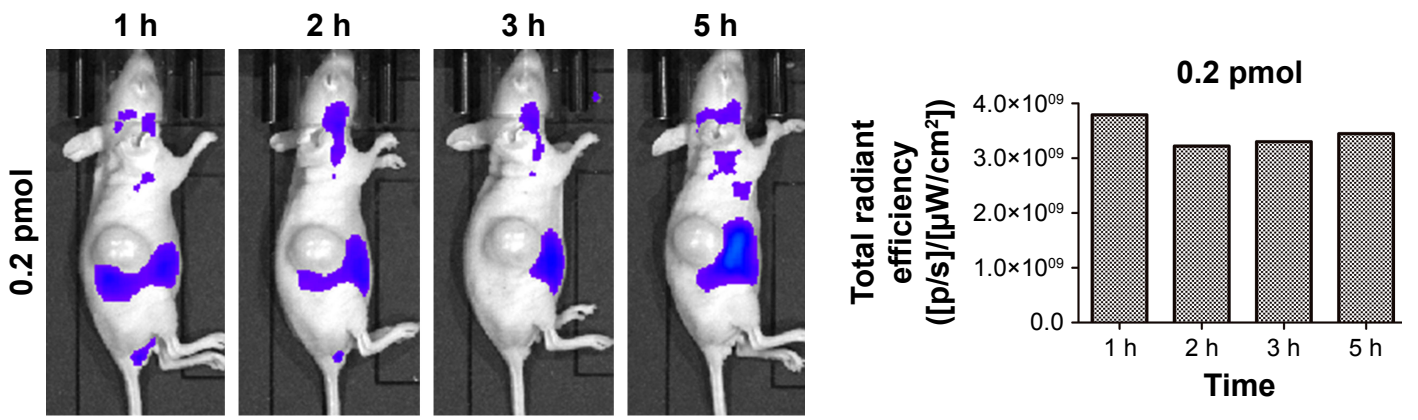

Epi-fluorescence
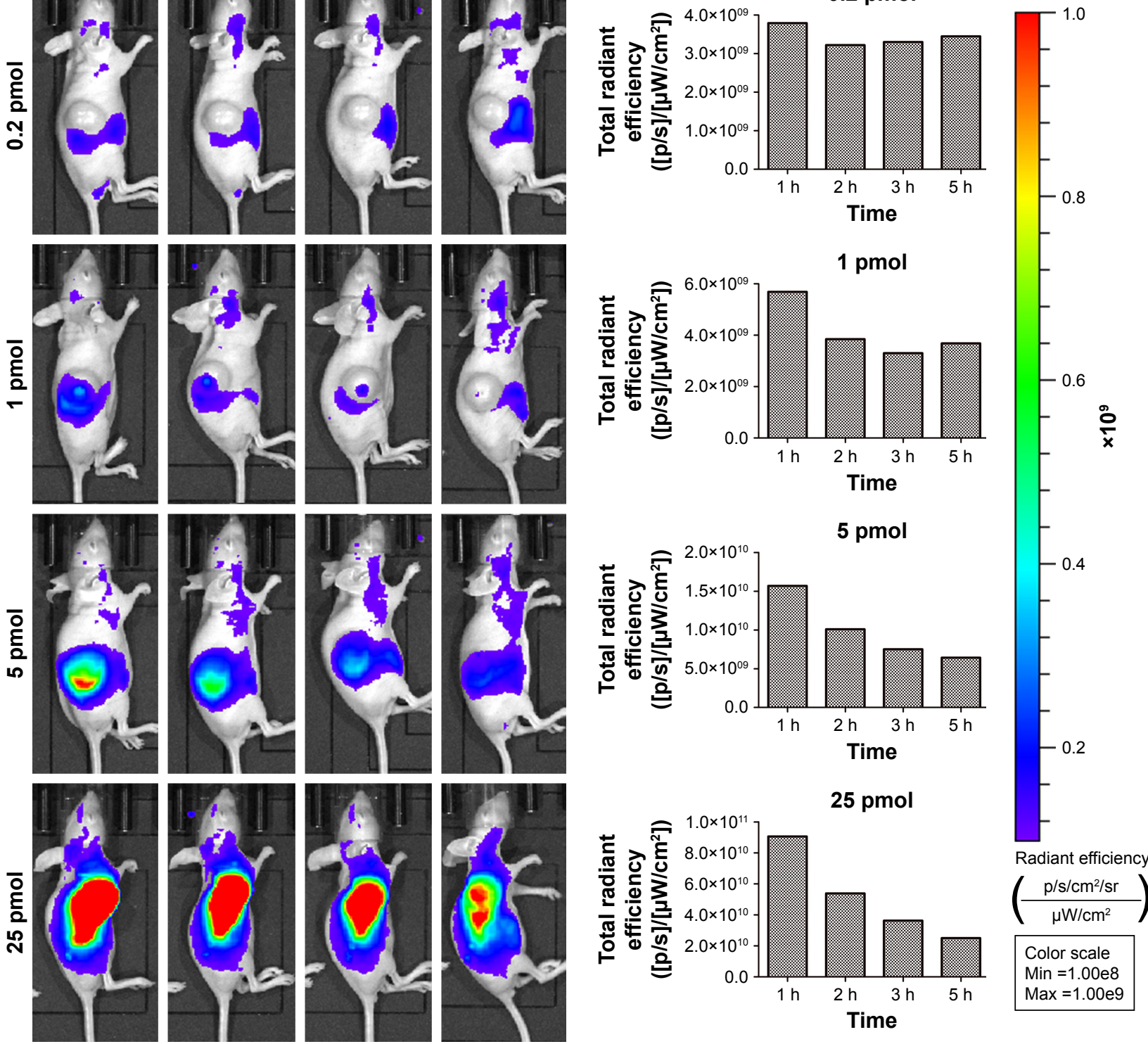

Radiant efficiency

$\left(\frac{\mathrm{p} / \mathrm{s} / \mathrm{cm}^{2} / \mathrm{sr}}{\mu \mathrm{W} / \mathrm{cm}^{2}}\right)$

Color scale

Min $=1.00 \mathrm{e} 8$ Max $=1.00 \mathrm{e} 9$

Figure 2 Temporal in vivo imaging of SWI990 pancreatic tumor-bearing mice intratumorally injected with different dosage of QDs-RGD.

Notes: Images were captured by IVIS Lumina XRMS III Imaging System. Each mouse was conducted by intratumoral injection. All images are acquired under the same instrumental conditions (eg, excitation: $610 \mathrm{~nm}$, exposure time: $1,000 \mathrm{~ms}$, emission wavelength: $705 \mathrm{~nm}$ ). Each image of mouse was conducted by the same color scale ( $\min =1.00 \mathrm{e} 8 ; \max =1.00 \mathrm{e} 9)$ and listed from left to right as time passed $(1,2,3$, and $5 \mathrm{~h}$ post injection). The data presented in the column diagram show the tendency of total radiant efficiency in the tumor area of each group as time passed.

Abbreviations: max, maximum; min, minimum; QDs, quantum dots; QDs-RGD, QDs conjugated with arginine-glycine-aspartic acid peptide sequence; RGD, arginineglycine-aspartic acid.

the optical fiber was inserted into a 20 gauge needle for the PDT procedure (Figure 4B). After intratumoral injection of 5 pmol of QDs-RGD, an optical fiber connected to a laser light was inserted directly into the tumors of mice in group 1 (Figure 4C). Mice in group 2 received an injection of QDs-RGD at the same dose, but the area around the tumor was irradiated (Figure 4D). In group 3, in regardless of the QD-RGD injection, the protocol was the same as for mice in group 1 . In the following 14 days, all mice showed no evidence of weakness, which meant that this treatment did not influence their safety. Then, PDT was done again for better inhibition of the tumor.

Figure 5 shows representative images of the tumor-bearing nude mice 1, 7, 14, 15, 21, and 28 days after light irradiation. The tumor volumes in groups 2 and 3 were much larger than those in group 1. For more detailed information, the $\mathrm{V} / \mathrm{V}_{0}$ of each group was collected in accordance with the number of days after treatment (Figure 6). In group 1, after 28 days, 


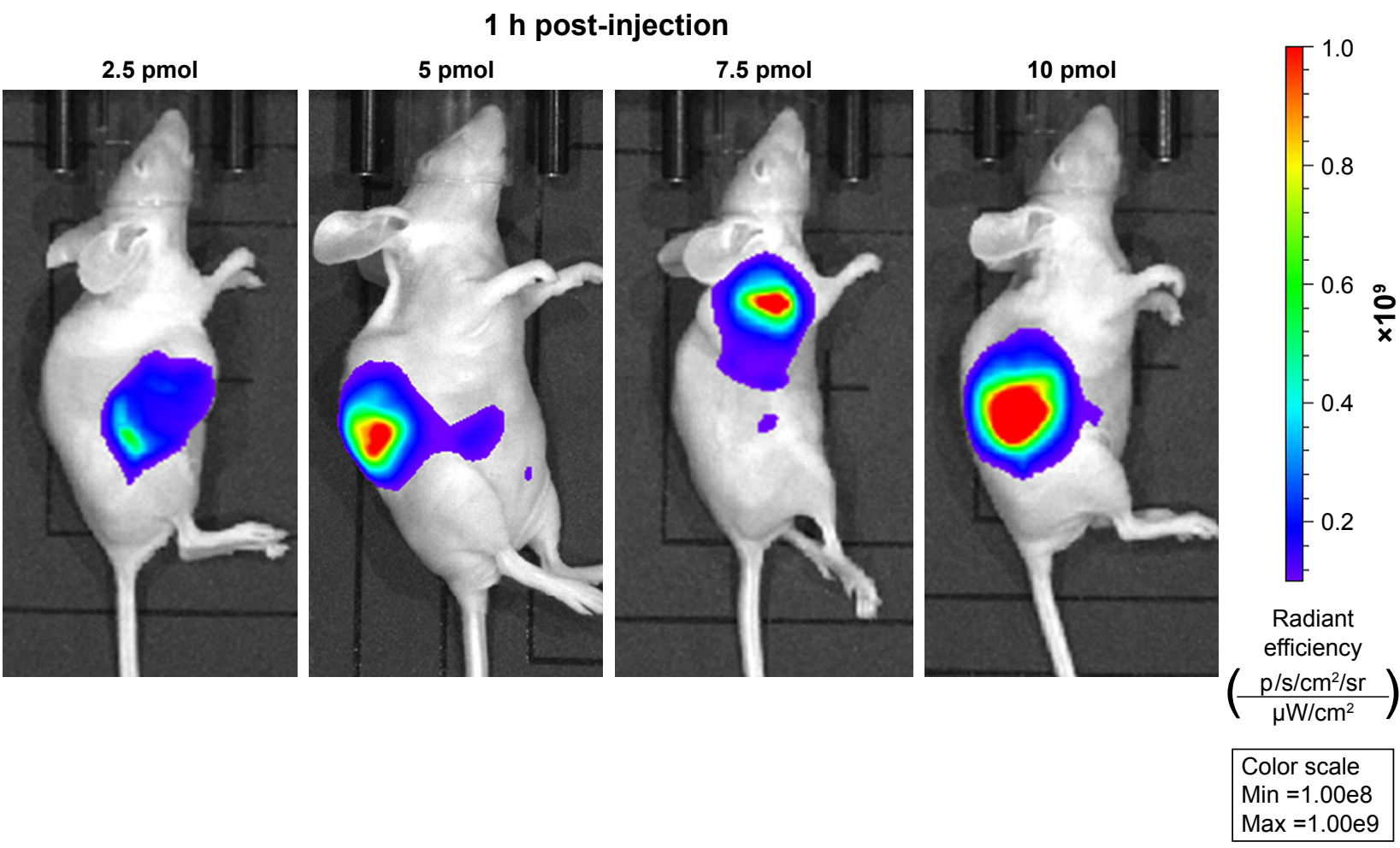

Figure 3 In vivo imaging of SWI990 pancreatic tumor-bearing mice I h post-intratumorally injected with different dosage of QDs-RGD.

Notes: Images were captured by IVIS Lumina XRMS III Imaging System. Each mouse was conducted by intratumoral injection. All images are acquired under the same instrumental conditions (eg, excitation: $610 \mathrm{~nm}$, exposure time: I,000 ms, emission wavelength: $705 \mathrm{~nm}$ ). Each image of mouse was conducted by the same color scale $(\min =1.00 \mathrm{e} 8 ; \max =1.00 \mathrm{e} 9)$ and listed from left to right according to different dosages $(2.5,5,7.5$, and 10 pmol).

Abbreviations: max, maximum; min, minimum; QDs, quantum dots; QDs-RGD, QDs conjugated with arginine-glycine-aspartic acid peptide sequence; RGD, arginineglycine-aspartic acid.
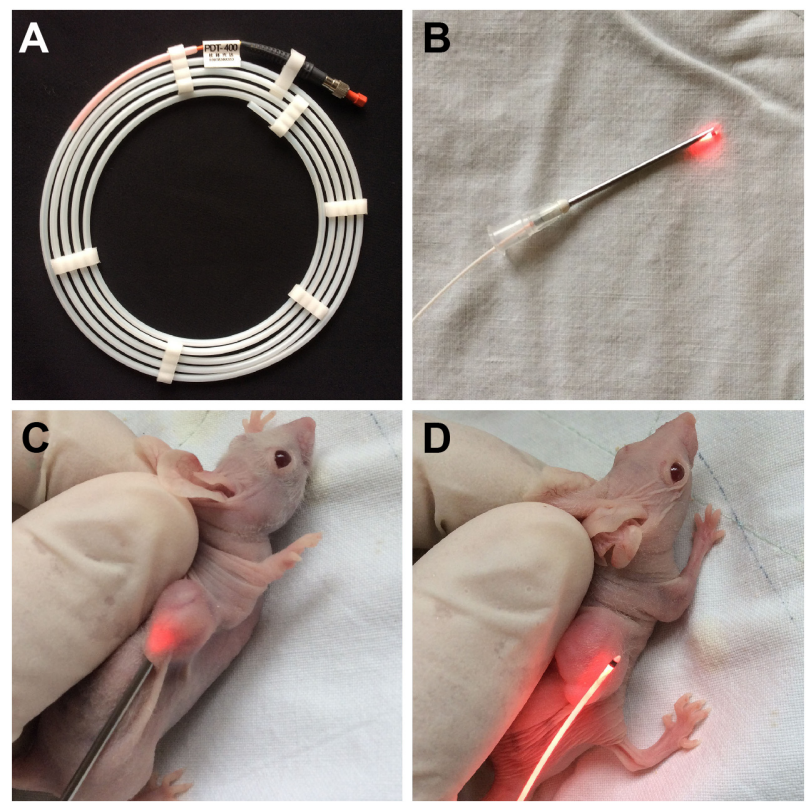

Figure 4 Camera image of laser optical fiber used in PDT and different PDT irradiating procedures performing on mice.

Notes: (A) Laser optical fiber; (B) fiber inserting into a needle for subsequent PDT treatment; (C) irradiating with fiber directly inserted into the tumor; and (D) irradiating surrounding around the tumor. Abbreviation: PDT, photodynamic therapy. tumors showed the distinct effects of tumor growth inhibition, with a mean $\mathrm{V} / \mathrm{V}_{0}$ ratio of $3.24 \pm 0.70$ (ie, the tumor grew to about threefold of its previous size). Moreover, tumors in the PDT group became septic after 21 days. However, mice in the control groups (which did not undergo QDs-RGD injection or did not receive direct irradiation) had rapid growth of the tumor, with a mean $\mathrm{V} / \mathrm{V}_{0}$ ratio of $6.08 \pm 0.50$ (group 2) and $7.25 \pm 0.82$ (group 3). The tumors in groups 2 and 3 grew significantly $(P<0.05)$ during the study period, suggesting that neither light irradiation around tumors nor an absence of QDs-RGD injection inhibited tumor growth.

\section{Histology}

The results of HE staining suggested that tumors in group 1 mice showed histologic abnormalities: notable inflammatory infiltrates with areas of suppurative necrosis and diffuse muscular alterations (Figure 7A). Apoptosis of cancer cell was not evident in group 2 or 3 . Conversely, TUNEL-positive cells were seen in tissue slices (Figure 7B). These results showed that PDT triggered damage to pancreatic cancer cells. After intratumoral injection of QDs-RGD and subsequent 


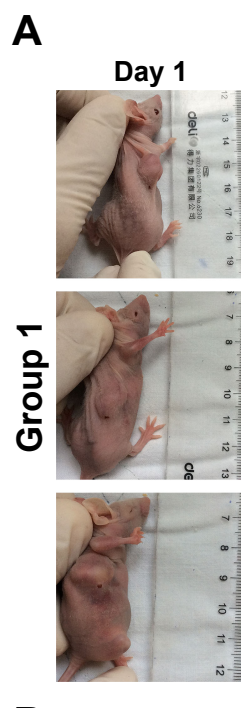

1st PDT

Day 7
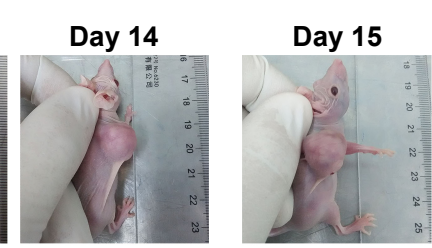

2nd PDT

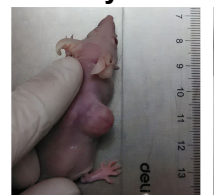

Day 21

\section{Day 28}
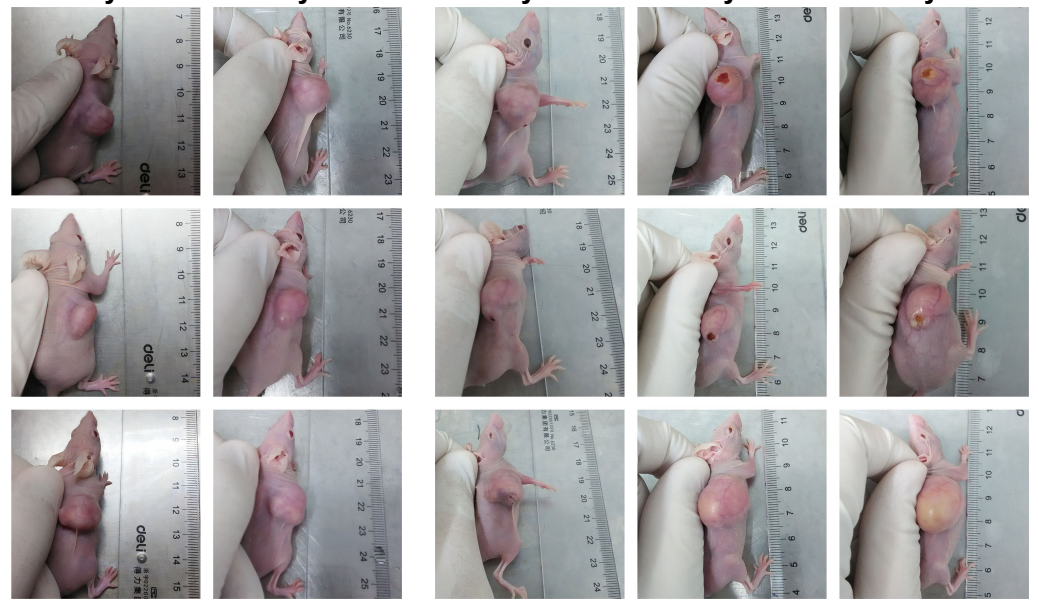

B

1st PDT

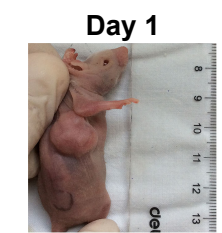

Day 7

Day 14
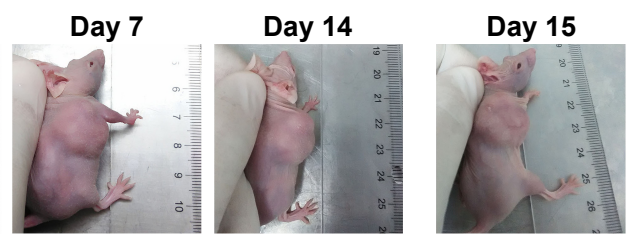

2nd PDT

Day 21

Day 28
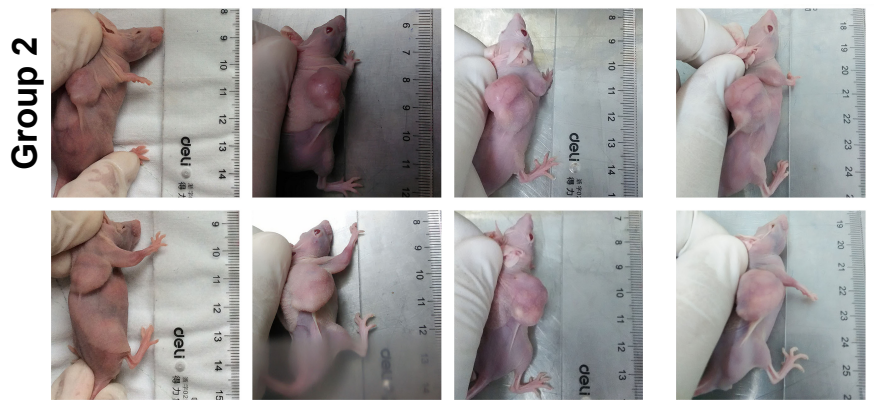

C

1st PDT

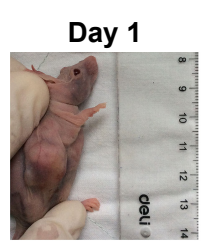

Day 7
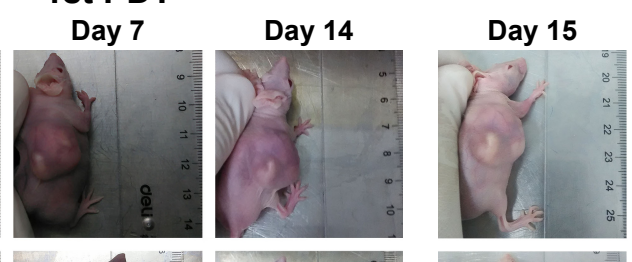

2nd PDT

Day 21
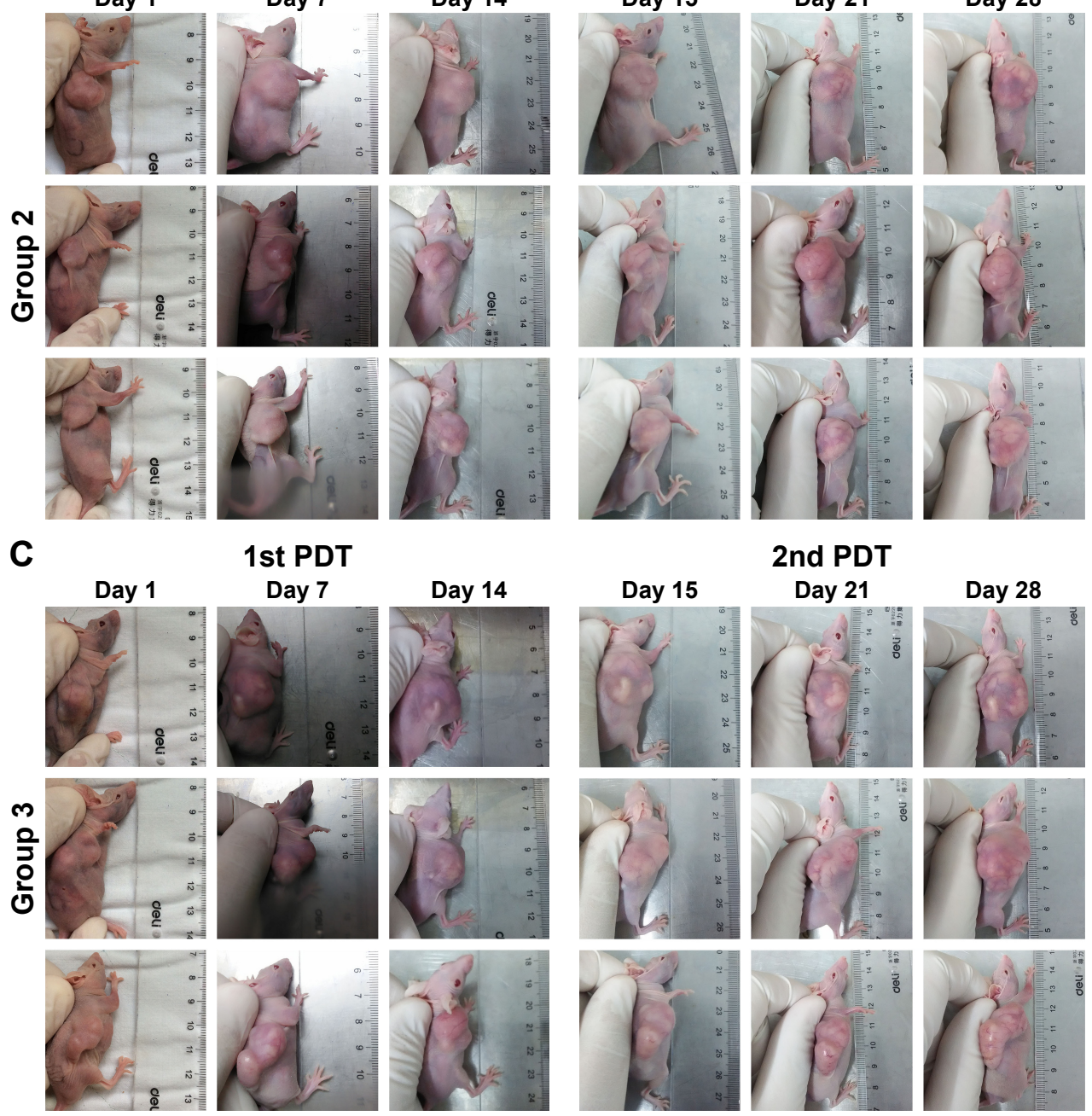

Figure 5 Photos of pancreatic tumor-bearing mouse after conducted with different PDT irradiating procedures.

Notes: (A) Group I, mice were intratumorally injected with 5 pmol of QDs-RGD and then irradiated with fiber (630 nm) directly inserted into the tumor for 20 min at a power density of $100 \mathrm{~mW} / \mathrm{cm}^{2} ;$ (B) group 2, mice received a QDs-RGD injection at the same dose, but the laser optical fiber was placed around, and not inserted into, tumors; (C) group 3, mice were irradiated without an injection of QDs-RGD, and the PDT procedure were conducted as same as group I. Two weeks later, the PDT procedure of every group was conducted once again.

Abbreviations: PDT, photodynamic therapy; QDs, quantum dots; QDs-RGD, QDs conjugated with arginine-glycine-aspartic acid peptide sequence; RGD, arginineglycine-aspartic acid. 


\section{A}

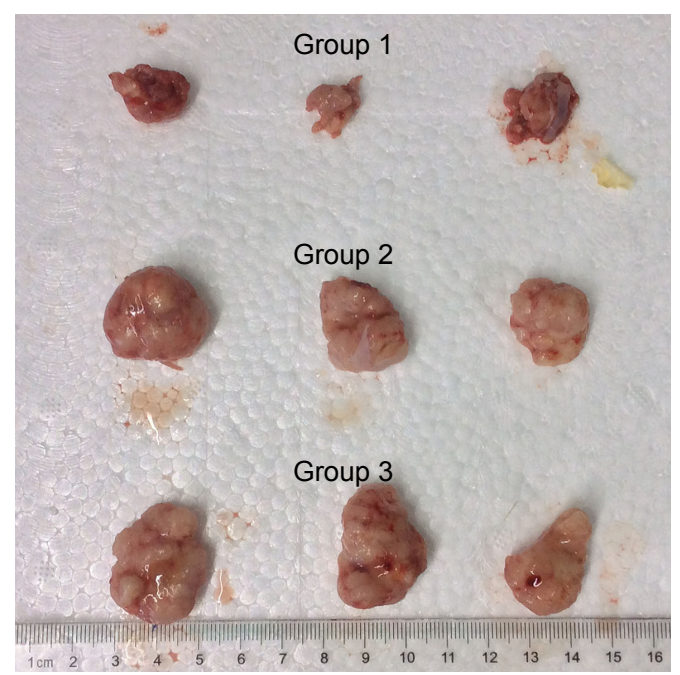

B

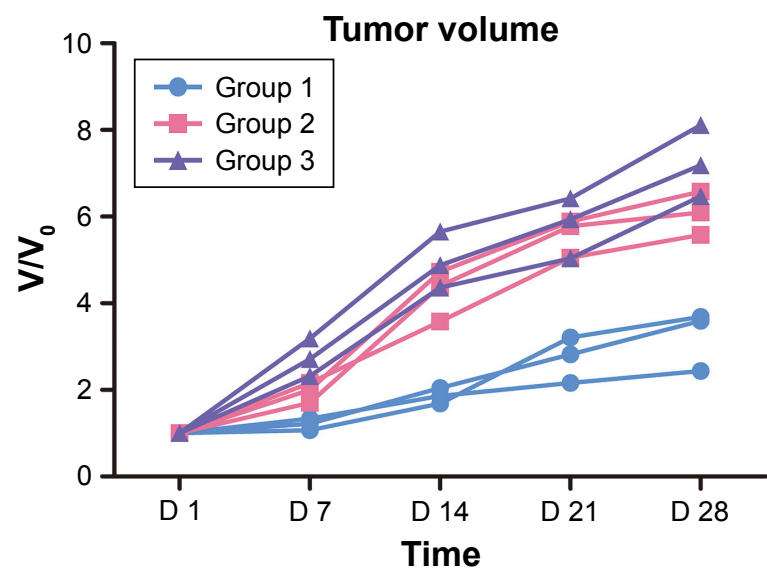

Figure 6 Efficacy of QDs-RGD-induced PDT treatment in nude mice bearing pancreatic neoplasm.

Notes: (A) Photo of dissected tumors in each group 28 days post-treatment. (B) Changes in the relative tumor volume ( $\mathrm{n}=3$ ) after different PDT procedures. At 28th day after PDT, the average $\mathrm{V} / \mathrm{V}_{0}$ ratio of group I reached to $3.24 \pm 0.70$, while the average $\mathrm{V} / \mathrm{V}_{0}$ ratios of groups 2 and 3 were $6.08 \pm 0.50$ and $7.25 \pm 0.82$. The tumors in the control groups were significantly $(P<0.05)$ larger than that in PDT group. Irradiating with fiber directly inserting into tumor showed an optimum therapeutic effect.

Abbreviations: PDT, photodynamic therapy; QDs, quantum dots; QDs-RGD, QDs conjugated with arginine-glycine-aspartic acid peptide sequence; RGD, arginineglycine-aspartic acid; $V / V_{0}$, tumor size divided by the previous tumor size; $\mathrm{D}$, day.

irradiation, multiple brown-stained spots were observed in the tissue sections of pancreatic tumors. Furthermore, we quantified the area of positively stained cells over the total area. With regard to the distribution of apoptotic cells under light $(\times 400$ magnification) microscopy, we took seven visual fields from each slice at random. In each field, we counted
200 cells and calculated the number of apoptotic cells: the mean value was $\approx 40 \%$.

\section{Discussion}

PDT has been regarded as a promising method for the treatment of various types of cancer and vascular proliferative
A

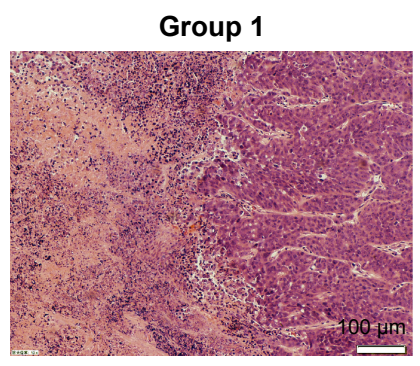

B

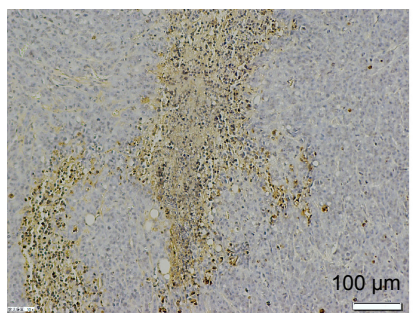

HE

Group 2

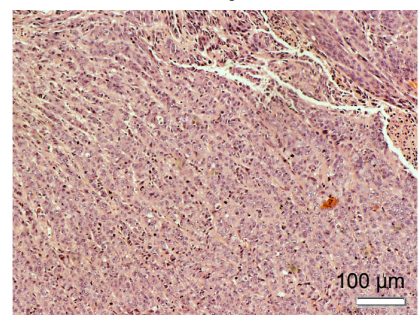

TUNEL

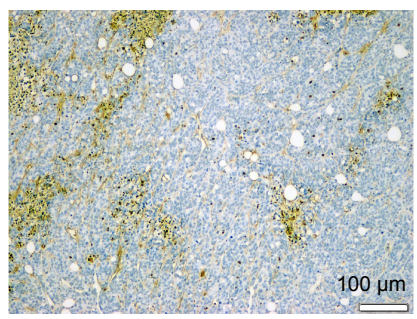

Group 3
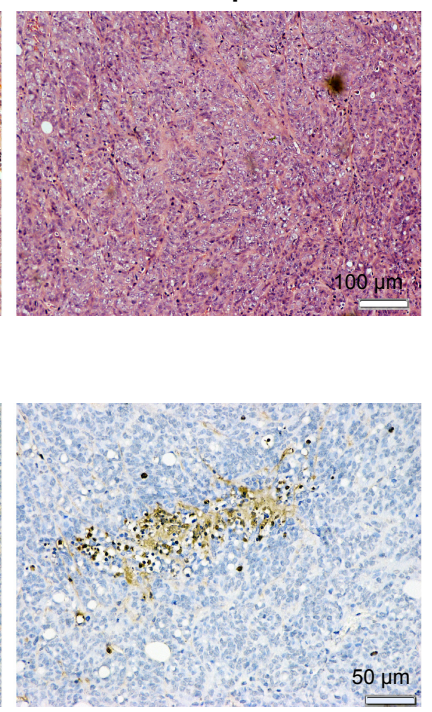

Figure $7 \mathrm{HE}$ and TUNEL staining micrograph of tumors from sacrificed mice.

Notes: (A) HE micrograph of tumor slices from three different groups. Necrosis tissues and inflammatory cells were detected in group I but hardly seen in group 2 or 3. The scale bar represents $100 \mu \mathrm{m}$. (B) TUNEL micrograph of tumor slice from group I. Multiple brown-stained cancer cells (TUNEL-positive cells) were observed in pancreatic tumor cells treated with irradiating by fiber directly inserting into tumor, indicating apoptosis. Scale bar: left $100 \mu \mathrm{m}$; middle $100 \mu \mathrm{m}$; right $50 \mu \mathrm{m}$.

Abbreviations: HE, hematoxylin and eosin; PDT, photodynamic therapy; TUNEL, terminal deoxynucleotidyl transferase (TdT)-mediated deoxyuridine triphosphate (dUTP)-biotin nick end labeling. 
diseases. ${ }^{38-40}$ Development of suitable photosensitizers is highly desired to improve PDT use. According to our studies, nanomaterials such as QDs-RGD have a PDT effect as novel photosensitizers for pancreatic cancer cells in vitro. Here, animal experiments were conducted to demonstrate PDT effects in vivo. However, some studies focusing on PDT in vivo could barely achieve efficient inhibition of tumor growth because only part of photosensitizers could reach tumors through the blood circulation. ${ }^{41-43}$ Most of the photosensitizers were distributed around the body and could induce injures to normal tissues. ${ }^{44}-47$ Those results should be attributed (at least in part) to the conventional intravenous mode of injection. In this way, more photosensitizers are used, but fewer work in the treatment. Hence, a new administration method, such as intratumoral injection, should be tried in PDT studies.

Another important aspect of intratumoral injection should be discussed. The pancreas lies deep within the abdomen, so insertion of drugs directly into its tissues by conventional methods is difficult. However, development of endoscopic ultrasonography (EUS) has enabled intratumoral injection of agents to pancreatic neoplasms. ${ }^{48-52}$ EUS has been used for more than three decades and has contributed considerably to the diagnosis and management of pancreatic cancer. Recently, the interventional applications of EUS for pancreatic disease (eg, pseudocyst drainage, control of pancreatic pain, ablation of cystic tumors, and solid tumor therapy) have been attempted. ${ }^{53-56} \mathrm{With}$ advancements in EUS-guided fine-needle aspiration (FNA) technology, EUS offers highresolution images of the pancreas. Hence, several types of drugs or local treatment modalities can be delivered directly into the pancreas. EUS-guided PDT can also reduce injection dosages and reduce the prevalence of adverse systemic side effects. ${ }^{57,58}$ Nanomaterials cannot be metabolized by the liver, so intratumoral injection of QDs-RGD would not influence their use for treatment. QDs-RGD might be the perfect photosensitizer for intratumoral injection of anticancer agents.

The first aim of our study was to discover an adequate dosage for PDT. Compared with the conventional intravenous injection, dosages used in this type of administration were much lower. Mice groups were sorted carefully in accordance with dosages $(0.2-25 \mathrm{pmol})$. According to comprehensive evaluation of all in vivo images, we chose the dosage group with relatively good fluorescence intensity $1 \mathrm{~h}$ post-injection. In the subsequent PDT, the irradiation procedure was done within $1 \mathrm{~h}$ to ensure that QDs-RGD did not disperse around the normal tissues of mice.
To obtain a better PDT effect on pancreatic tumor cells, the irradiation procedure was improved. If a laser optical fiber is inserted directly into tumors (eg, as in EUS-FNA treatments), the photosensitizer might produce more ROS and kill more tumor cells because little luminous energy would be wasted by skin penetration. We created a PDT group to demonstrate this hypothesis whereby irradiation was done by insertion of a laser optical fiber directly into the tumor. Control groups were set to investigate each part of the PDT procedure. Group 2 focused on the irradiation procedure (insertion into tumors or not), whereas group 3 was based on the photosensitizer (with or without QDs-RGD). After 28 days, the mean tumor volume of group 1 was much smaller than that of the control groups. The $\mathrm{V} / \mathrm{V}_{0}$ ratios could demonstrate the robustness of our hypothesis. Histology showed that if the irradiation procedure was done by EUS-FNA, inhibition of tumor growth by PDT would be much better.

In our work, nude mice were employed because they can be used to cultivate heterogeneous neoplasms with the aid of immunosuppression. SW1990 cells $\left(5 \times 10^{6}\right)$ were injected (sc) with $200 \mu \mathrm{L}$ PBS on to the back of nude mice. After 28 days, the tumor size reached $55-100 \mathrm{~mm}^{3}$, which could be used for assessment of the PDT effect of QDs-RGD. In the future, we will undertake experiments in immune-competent mice and mouse pancreatic cancer cells.

For future use in humans, intratumoral injection of QDs-RGD implies precise localization of the treatment area and a method of application that limits the diffusion of photosensitizers to pathologic areas. This strategy could be feasible with the help of EUS. Pancreatic neoplasms lying deep in the abdomen could be detected and targeted precisely using endoscopic methods to localize them. Taken together, our results suggest that intratumoral injection of QDs-RGD for PDT of pancreatic cancers in humans could be feasible. Evaluation of the efficacy and potential advantages of local use of QDs-RGD-PDT (eg, avoidance of incorrect penetration or better access of the photosensitizing agent) will require specific, dedicated studies.

\section{Conclusion}

QDs-RGD, which are novel semiconductor nanoparticles with feasible optical properties for PDT, were used as photosensitizers in mice bearing pancreatic neoplasms. In this experimental model, PDT coupled with intratumoral injection of QDs-RGD and illumination of fibers inserted directly into tumors appeared to be efficacious for local management of pancreatic neoplasms. QDs-RGD are appropriate photosensitizers, and further studies in humans are required 
to assess their efficacy in vivo for the treatment of such inoperable patients.

\section{Acknowledgments}

This study was supported by the National Natural Science Foundation of China (grant number 81472844) and Shanghai Municipal Education Commission (14ZZ114). We thank Min Zhou for assistance with the collection and analyses of data. Li MM and Cao J share co-first authorship.

\section{Author contributions}

Li MM and Cao J contributed equally to this study. Li MM and Cao $\mathrm{J}$ undertook most of the experiments and drafted the manuscript; Cao J and $\mathrm{Xu}$ LM designed the study and supervised its implementation; Yang JC, Shen YJ, Chen YW, Qu CY, Zhang Y, and Shen F participated in the experiments; Cai XL analyzed the data; all authors made critical revisions and approved the final version of the manuscript. All authors contributed toward data analysis, drafting and critically revising the paper and agree to be accountable for all aspects of the work.

\section{Disclosure}

The authors report no conflicts of interest in this work.

\section{References}

1. Wolfgang CL, Herman JM, Laheru DA, et al. Recent progress in pancreatic cancer. CA Cancer J Clin. 2013;63(5):318-348.

2. Hussain SP. Pancreatic cancer: current progress and future challenges. Int J Biol Sci. 2016;12(3):270-272.

3. Kamisawa T, Wood LD, Itoi T, Takaori K. Pancreatic cancer. Lancet. 2016;388(10039):73-85.

4. Anderson B, Karmali S. Laparoscopic resection of pancreatic adenocarcinoma: dream or reality? World J Gastroenterol. 2014;20(39): 14255-14262.

5. Hackert T, Ulrich A, Buchler MW. Borderline resectable pancreatic cancer. Cancer Lett. 2016;375(2):231-237.

6. Shaib WL, Ip A, Cardona K, et al. Contemporary management of borderline resectable and locally advanced unresectable pancreatic cancer. Oncologist. 2016;21(2):178-187.

7. Gresham GK, Wells GA, Gill S, Cameron C, Jonker DJ. Chemotherapy regimens for advanced pancreatic cancer: a systematic review and network meta-analysis. BMC Cancer. 2014;14:471

8. Hajj C, Goodman KA. Role of radiotherapy and newer techniques in the treatment of GI cancers. J Clin Oncol. 2015;33(16):1737-1744.

9. Silvestris N, Gnoni A, Brunetti AE, et al. Target therapies in pancreatic carcinoma. Curr Med Chem. 2014;21(8):948-965.

10. Mallidi S, Anbil S, Bulin AL, Obaid G, Ichikawa M, Hasan T. Beyond the barriers of light penetration: strategies, perspectives and possibilities for photodynamic therapy. Theranostics. 2016;6(13):2458-2487.

11. Saboktakin MR, Tabatabaee RM. The novel polymeric systems for photodynamic therapy technique. Int J Biol Macromol. 2014;65: 398-414.

12. Garg T, Jain NK, Rath G, Goyal AK. Nanotechnology-based photodynamic therapy: concepts, advances, and perspectives. Crit Rev Ther Drug Carrier Syst. 2015;32(5):389-439.
13. Oniszczuk A, Wojtunik-Kulesza KA, Oniszczuk T, Kasprzak K. The potential of photodynamic therapy (PDT)-experimental investigations and clinical use. Biomed Pharmacother. 2016;83:912-929.

14. Abrahamse H, Hamblin MR. New photosensitizers for photodynamic therapy. Biochem J. 2016;473(4):347-364.

15. Li JW, Wu ZM, Magetic D, Zhang LJ, Chen ZL. Antitumor effects evaluation of a novel porphyrin derivative in photodynamic therapy. Tumour Biol. 2015;36(12):9685-9692.

16. Ge J, Lan M, Zhou B, et al. A graphene quantum dot photodynamic therapy agent with high singlet oxygen generation. Nat Commun. 2014; 5:4596.

17. Cy Hyun Kim CH, Chung Wook Chung CW, Hye Myeong Lee HM, et al. Synergistic effects of 5-aminolevulinic acid based photodynamic therapy and celecoxib via oxidative stress in human cholangiocarcinoma cells. Int J Nanomedicine. 2013;8:2173-2186.

18. Bhatta AK, Keyal U, Wang XL. Photodynamic therapy for onychomycosis: a systematic review. Chem Soc Rev. 2016;15:228-235.

19. Abd-Elgaliel WR, Cruz-Monserrate Z, Wang H, Logsdon CD, Tung CH. Pancreatic cancer-associated Cathepsin $\mathrm{E}$ as a drug activator. $J$ Control Release. 2013;167(3):221-227.

20. Broekgaarden M, Weijer R, van Gulik TM, Hamblin MR, Heger M. Tumor cell survival pathways activated by photodynamic therapy: a molecular basis for pharmacological inhibition strategies. Cancer Metastasis Rev. 2015;34(4):643-690.

21. Alexiou A, Vairaktarakis C, Tsiamis V, Ashraf GM. Application of efficient nanoparticles for early diagnosis and treatment of cancer. Curr Drug Metab. 2015;16(8):662-675.

22. Cuenca AG, Jiang H, Hochwald SN, Delano M, Cance WG, Grobmyer SR. Emerging implications of nanotechnology on cancer diagnostics and therapeutics. Cancer. 2006;107(3):459-466.

23. Liu Q, Li H, Xia Q, Liu Y, Xiao K. Role of surface charge in determining the biological effects of $\mathrm{CdSe} / \mathrm{ZnS}$ quantum dots. Int J Nanomedicine. 2015;10:7073-7088.

24. Tomczak N, Liu R, Vancso JG. Polymer-coated quantum dots. Nanoscale. 2013;5(24):12018-12032.

25. Hsu CY, Chen CW, Yu HP, Lin YF, Lai PS. Bioluminescence resonance energy transfer using luciferase-immobilized quantum dots for self-illuminated photodynamic therapy. Biomaterials. 2013;34(4): 1204-1212.

26. Colombeau L, Acherar S, Baros F, et al. Inorganic nanoparticles for photodynamic therapy. In: Sortino S, editor. Light-Responsive Nanostructured Systems for Applications in Nanomedicine. Cham: Springer International Publishing; 2016:113-134.

27. Das A, Snee PT. Synthetic developments of nontoxic quantum dots. Chemphyschem. 2016;17(5):598-617.

28. Karakoti AS, Shukla R, Shanker R, Singh S. Surface functionalization of quantum dots for biological applications. Adv Colloid Interface Sci. 2015;215:28-45.

29. Lin G, Wang X, Yin F, Yong KT. Passive tumor targeting and imaging by using mercaptosuccinic acid-coated near-infrared quantum dots. Int J Nanomedicine. 2015;10:335-345.

30. Arruda Macedo JK, Fox JW, de Souza Castro M. Disintegrins from snake venoms and their applications in cancer research and therapy. Curr Protein Pept Sci. 2015;16(6):532-548.

31. Chakravarty R, Chakraborty S, Dash A. Molecular imaging of breast cancer: role of RGD peptides. Mini Rev Med Chem. 2015;15(13): 1073-1094.

32. Liu X, Cui W, Li B, Hong Z. Targeted therapy for glioma using cyclic RGD-entrapped polyionic complex nanomicelles. Int J Nanomedicine. 2012;7:2853-2862.

33. He SJ, Cao J, Li YS, et al. CdSe/ZnS quantum dots induce photodynamic effects and cytotoxicity in pancreatic cancer cells. World $J$ Gastroenterol. 2016;22(21):5012-5022.

34. Zhou M, Ni QW, Yang SY, et al. Effects of integrin-targeted photodynamic therapy on pancreatic carcinoma cell. World J Gastroenterol. 2013;19(39):6559-6567. 
35. Obstoy B, Salaun M, Bohn P, Veresezan L, Sesboué R, Thiberville L. Photodynamic therapy using methylene blue in lung adenocarcinoma xenograft and hamster cheek pouch induced squamous cell carcinoma. Photodiagnosis Photodyn Ther. 2016;15:109-114.

36. Wei J, Luo X, Chen M, Lu J, Li X. Spatial distribution and antitumor activities after intratumoral injection of fragmented fibers with loaded hydroxycamptothecin. Acta Biomater. 2015;23:189-200.

37. Green H, Crockett S, Martyshkin D, et al. A histological evaluation and in vivo assessment of intratumoral near infrared photothermal nanotherapy-induced tumor regression. Int J Nanomedicine. 2014;9: 5093-5102.

38. Middelkamp-Hup MA, Sanchez-Carpintero I, Kossodo S, et al. Photodynamic therapy for cutaneous proliferative vascular tumors in a mouse model. J Invest Dermatol. 2003;121(3):634-639.

39. Hu J, Tang Y, Elmenoufy AH, Xu H, Cheng Z, Yang X. Nanocompositebased photodynamic therapy strategies for deep tumor treatment. Small. 2015;11(44):5860-5887.

40. Rao N, Sunkara M, Amreddy N, Kurra V, Adimoolam M. Photosensitizer and peptide-conjugated PAMAM dendrimer for targeted in vivo photodynamic therapy. Int J Nanomedicine. 2015;10:6865-6878.

41. Li PX, Mu JH, Xiao HL, Li DH. Antitumor effect of photodynamic therapy with a novel targeted photosensitizer on cervical carcinoma. Oncol Rep. 2015;33(1):125-132.

42. Zou Q, Zhao H, Zhao Y, et al. Effective two-photon excited photodynamic therapy of xenograft tumors sensitized by water-soluble bis(arylidene)cycloalkanone photosensitizers. J Med Chem. 2015; 58(20):7949-7958

43. Song X, Zhang R, Liang C, Chen Q, Gong H, Liu Z. Nano-assemblies of J-aggregates based on a NIR dye as a multifunctional drug carrier for combination cancer therapy. Biomaterials. 2015;57:84-92.

44. Wang YW, Yang K, Tang H, Chen D, Bai YL. Toxicity assessment of repeated intravenous injections of arginine-glycine-aspartic acid peptide conjugated $\mathrm{CdSeTe} / \mathrm{ZnS}$ quantum dots in mice. Int J Nanomedicine. 2014;9:4809-4817.

45. Rocha LB, Schaberle F, Dabrowski JM, Simoes S, Arnaut LG. Intravenous single-dose toxicity of redaporfin-based photodynamic therapy in rodents. Int J Mol Sci. 2015;16(12):29236-29249.

46. Gamal-Eldeen AM, Fouad LM, El-Daly SM, El-Hussieny EA, El Denshary ES. Photodynamic therapeutic role of indocyanine green in tumor-associated inflammation in skin cancer. Photodiagnosis Photodyn Ther. 2014;11(2):239-249.

47. Wang M, Wang J, Sun H, et al. Time-dependent toxicity of cadmium telluride quantum dots on liver and kidneys in mice: histopathological changes with elevated free cadmium ions and hydroxyl radicals. Int $J$ Nanomedicine. 2016;11:2319-2328.
48. De Lisi S, Giovannini M. Endoscopic ultrasonography: transition towards the future of gastro-intestinal diseases. World J Gastroenterol. 2016; 22(5):1779-1786.

49. Hecht JR, Bedford R, Abbruzzese JL, et al. A phase I/II trial of intratumoral endoscopic ultrasound injection of ONYX-015 with intravenous gemcitabine in unresectable pancreatic carcinoma. Clin Cancer Res. 2003;9(2):555-561.

50. Kitano M, Kamata K, Imai H, et al. Contrast-enhanced harmonic endoscopic ultrasonography for pancreatobiliary diseases. Dig Endosc. 2015; 27(suppl 1):60-67.

51. Suzuki R, Thosani N, Annangi S, Guha S, Bhutani MS. Diagnostic yield of EUS-FNA-based cytology distinguishing malignant and benign IPMNs: a systematic review and meta-analysis. Pancreatology. 2014; 14(5):380-384.

52. Hecht JR, Farrell JJ, Senzer N, et al. EUS or percutaneously guided intratumoral TNFerade biologic with 5-fluorouracil and radiotherapy for first-line treatment of locally advanced pancreatic cancer: a phase I/II study. Gastrointest Endosc. 2012;75(2):332-338.

53. Paik WH, Seo DW, Dhir V, Wang HP. Safety and efficacy of EUSguided ethanol ablation for treating small solid pancreatic neoplasm. Medicine. 2016;95(4):e2538.

54. Saul A, Luna MAR, Chan C, et al. EUS-guided drainage of pancreatic pseudocysts offers similar success and complications compared to surgical treatment but with a lower cost. Surg Endosc. 2016;30(4) 1459-1465.

55. Rustagi T, Gleeson FC, Chari ST, et al. Endoscopic ultrasound fine needle aspiration diagnosis of synchronous primary pancreatic adenocarcinoma and effects on staging and resectability. Clin Gastroenterol Hepatol. 2017;15(2):299.e-302.e.

56. Villa NA, Berzosa M, Wallace MB, Raijman I. Endoscopic ultrasoundguided fine needle aspiration: the wet suction technique. Endosc Ultrasound. 2016;5(1):17-20.

57. Yusuf TE, Matthes K, Brugge WR. EUS-guided photodynamic therapy with verteporfin for ablation of normal pancreatic tissue: a pilot study in a porcine model (with video). Gastrointest Endosc. 2008; 67(6):957-961.

58. Choi JH, Oh D, Lee $\mathrm{JH}$, et al. Initial human experience of endoscopic ultrasound-guided photodynamic therapy with a novel photosensitizer and a flexible laser-light catheter. Endoscopy. 2015;47(11) 1035-1038.
International Journal of Nanomedicine

\section{Publish your work in this journal}

The International Journal of Nanomedicine is an international, peerreviewed journal focusing on the application of nanotechnology in diagnostics, therapeutics, and drug delivery systems throughout the biomedical field. This journal is indexed on PubMed Central,

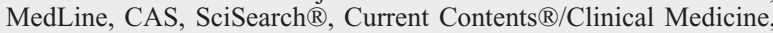

\section{Dovepress}

Journal Citation Reports/Science Edition, EMBase, Scopus and the Elsevier Bibliographic databases. The manuscript management system is completely online and includes a very quick and fair peer-review system, which is all easy to use. Visit http://www.dovepress.com/ testimonials.php to read real quotes from published authors. 